EDITORIAL

\title{
PREANALYTICAL VARIABLES AND LABORATORY PERFORMANCE
}

\author{
Praveen Sharma* \\ Department of Biochemistry, SMS Medical College, Jaipur, India
}

Radical changes have occurred in the organization, number and type of tests and in the role of medical laboratories in healthcare over recent years. The affirmation of a new role for laboratory professionals calls for greater analytical accuracy and more stringent test selection and interpretation of results. The question remains, however, as to where the greatest needs for improvement to achieve these goals are. Laboratory data are an integral, often pivotal, part of the complex decisionmaking process, influencing up to $70 \%$ of medical diagnoses. The increasing awareness of issues involving medical errors within healthcare has cast a spotlight on the factors that contribute to the resulting adverse events and has also made clinical laboratories the subject of scrutiny as essential parts of the overall healthcare system. Therefore, additional sources of variation in the entire laboratory testing process should become the focus for further and necessary quality improvements. Errors occurring within the extra-analytical phases are still the prevailing source of concern.

As preanalytical sources of variation can produce unpredictable and unfavorable impacts on the wellbeing of patients, a reduction in laboratory testing errors and quality improvements both play a significant role in programs for assessing and improving quality in healthcare. By definition, technology is dynamic and in many ways it dictates advances in laboratory diagnostics. Automation, databases and computers have greatly simplified many aspects of previously tedious tasks, creating a greater volume of routine work, as well as significantly improving the analytical error rate over time. Therefore, mistakes outside the analytical phase of testing seem more likely to affect the usefulness of laboratory results in patient care. The attention of laboratory professionals should now be focused on alternative and prevailing sources

\section{Address for Correspondence :}

Prof. Praveen Sharma,

Department of Biochemistry,

SMS Medical College, Jaipur-302004

M: 91-9414044562

E-mail: praveensharma55@gmail.com of errors, such as those occurring within the preanalytical and post-analytical phases.

There are some further and less controllable sources of preanalytical errors that can seriously influence the reliability of laboratory testing, but which are barely identifiable by laboratory staff. These primarily include patient-related physical variables (physical exercise, diet, stress, positional effects, menstruation, pregnancy, life style, age, sex, season altitude), mild or visually undetectable hemolysis, hemolyzed specimens for analyses that do not require sample separation, and prolonged tourniquet stasis during blood drawing. Owing to regular training-induced variations of plasma volume and metabolites, regular physical exercise has a strong influence on several biochemical and hematological variables. Therefore, interpretation of some laboratory data in physically active individuals may require caution, as results falling outside the conventional reference ranges are more likely to reflect a physiological adaptation to regular training rather than underlying pathologies. Thus, individual lifestyle and biological rhythms should be always taken into consideration before sample collection.

The preparation of subjects for blood specimen collection such as the duration of overnight fast, time of specimen collection, duration of tourniquet application, infusion, effect of anticoagulants and stabilizing additives, specimen handling and processing like test requests, patient identification and Sample labelling, specimen collection volume, transport of blood samples, centrifugation, storage time and temperature, need to be delineated and standardized to minimize preanalytical errors. Preanalytical errors can contribute to 32$75 \%$ of total laboratory errors while analytical errors remain $13-32 \%$. From a theoretical viewpoint, the pre-analytical phase can be further subdivided into two parts: in one, the so called "pre-preanalytical phase", the clinicians decides which laboratory test should be performed on the basis of his/her knowledge and experience; the other, the "conventional" preanalytical phase, involves a series of related processes starting from patient identification, through the choice of right collection tubes and ending with transportation and preparation for 
analysis of the samples.

\section{Who Is Involved in Preanalytics?}

There are always several persons involved in Preanalytics: The patient, the physician, the nurse, the attendant, the transport service, the medical technical assistant and the laboratory doctor; they all share the responsibility for the quality of the specimen material and should understand the significance of the preanalytical phase, as well as recognize possible causes of error and their consequence for the examination results.

Activities during and after the preanalytical phase and responsible personnel

\begin{tabular}{|c|c|}
\hline Activities & Persons involved \\
\hline Requisition for analysis & Physician \\
\hline Preparation of patient & $\begin{array}{l}\text { Physician, nursing staff, } \\
\text { doctor's assistant, patient }\end{array}$ \\
\hline $\begin{array}{l}\text { Identification of patients } \\
\text { and specimens }\end{array}$ & $\begin{array}{l}\text { Physician, nursing staff, } \\
\text { doctor's assistant, patient }\end{array}$ \\
\hline Blood collection & $\begin{array}{l}\text { Physician, nursing staff, doctor's } \\
\text { assistant }\end{array}$ \\
\hline Mixing with anticoagulants & $\begin{array}{l}\text { Physician, nursing staff, doctor's } \\
\text { assistant }\end{array}$ \\
\hline Storage until transportation & Nursing staff, doctor's assistant \\
\hline Transportation & Collection or courier service \\
\hline $\begin{array}{l}\text { Acceptance, storage and } \\
\text { preparation of samples }\end{array}$ & $\begin{array}{l}\text { Laboratory staff, medical technical } \\
\text { assistants, laboratory experts }\end{array}$ \\
\hline
\end{tabular}

The time required for the preanalytical phase is often underestimated. In fact, it takes up more than $58 \%$ of total time required, which is more than required for the laboratory analysis. With the available modern technology, the actual analysis only takes up around $25 \%$ of the time.

It is now widely accepted that spurious changes in laboratory testing arising from inappropriate or inaccurate application of rigid preanalytical protocols might be harmful and misleading, consuming valuable healthcare resources and leading to potential errors or delays in patient care. As laboratory testing errors mainly occur outside the analytical process, they are likely to span the current branches or subspecialties of laboratory medicine, including clinical biochemistry, hematology, coagulation, immunometry and molecular biology. Inappropriate laboratory utilization ultimately increases healthcare costs, harms patients and perpetuates the vision of laboratory testing as a commodity. Laboratory expenditure as a proportion of total hospital care accounts for $4 \%$ in the United Kingdom, 5.2\% in Australia, 710\% in Canada and 5\% in the United States. However, no such data are available for developing countries like India. Improvements in specimen quality and result utilization are hence essential for quality improvement initiatives and health cost reductions.

Competent knowledge of these possible sources of variability is a critical precondition for their avoidance, although there is nearly universal agreement that laboratory tests are overutilized and one of the biggest sources of preanalytical variability might be differences in the test-ordering patterns of care providers. Attempts to reduce unnecessary testing have often been difficult to implement or sustain. Interventions with the greatest impact use multiple approaches, are repeated regularly and include multifaceted education, peer assessment and effective feedback strategies. Besides direct policies aimed at improving the appropriateness of test requests and utilization, additional preanalytical factors are common causes of inaccurate test results. Moreover, most complaints about unreliable laboratory testing are frequently a direct outcome of incorrect techniques immediately associated with the sampling procedure, such as phlebotomy and specimen collection. In this respect, consistent quality specimens, resulting from proper training and knowledge of the factors that can influence laboratory results, are essential for minimizing errors and optimizing resource utilization and quality and finally improving the whole patient management process. The pursuit of safety is a multidisciplinary enterprise. This is as true for patient safety, hitherto an exclusively medical domain, as for any other safety issue.

It is thus clear that human error in medicine does exist and is a profound challenge. As is necessary when seeking to solve any problem, the problem must first be described and the factors contributing to it be identified. Given the nature of humans to err, we are aware that the complete elimination of laboratory testing errors is unrealistic, especially those relating to extra-analytical phases that are harder to control, highlights the importance of good laboratory practice and compliance with the new accreditation standards, which encompass the adoption of suitable strategies for error prevention, tracking and reduction, including process redesign, the use of extraanalytical specifications and improved communication among care-givers.

${ }^{*}$ Author is Editor-in-Chief, Indian Journal of Clinical Biochemistry. 